\title{
Comparison of Semidefinite Relaxation Detectors for High-Order Modulation MIMO Systems
}

\author{
Z. Y. Shao, ${ }^{1}$ S. W. Cheung, ${ }^{2}$ and T. I. Yuk ${ }^{2}$ \\ ${ }^{1}$ School of Mechanical \& Electric Engineering, Guangzhou University, Guangzhou Higher Education Mega Center, \\ Guangzhou 510006, China \\ ${ }^{2}$ Department of Electrical \& Electronic Engineering, University of Hong Kong, Pokfulam Road, Hong Kong
}

Correspondence should be addressed to Z. Y. Shao; zyshao@eee.hku.hk

Received 30 June 2014; Revised 26 October 2014; Accepted 30 October 2014; Published 27 November 2014

Academic Editor: Stefano Selleri

Copyright (c) 2014 Z. Y. Shao et al. This is an open access article distributed under the Creative Commons Attribution License, which permits unrestricted use, distribution, and reproduction in any medium, provided the original work is properly cited.

\begin{abstract}
Multiple-input multiple-output (MIMO) system is considered to be one of the key technologies of LTE since it achieves requirements of high throughput and spectral efficiency. The semidefinite relaxation (SDR) detection for MIMO systems is an attractive alternative to the optimum maximum likelihood (ML) decoding because it is very computationally efficient. We propose a new SDR detector for 256-QAM MIMO system and compare its performance with two other SDR detectors, namely, BC-SDR detector and VA-SDR detector. The tightness and complexity of these three SDR detectors are analyzed. Both theoretical analysis and simulation results demonstrate that the proposed SDR can provide the best BLER performance among the three detectors, while the BC-SDR detector and the VA-SDR detector provide identical BLER performance. Moreover, the BC-SDR has the lowest computational complexity and the VA-SDR has the highest computational complexity, while the proposed SDR is in between.
\end{abstract}

\section{Introduction}

Multiple-input multiple-output (MIMO) system has been considered as a promising solution to provide high data rate and good quality of future wireless communications. In MIMO systems, detection algorithm is one of the major challenges due to its limitations of either unsatisfactory performance or high complexity. The maximum likelihood (ML) detection can provide the best block-error-rate (BLER) performance, but its computational complexity is extremely high since it searches the vectors in the entire lattice space of the transmitted signals. Although equalization-based detectors such as zero-forcing (ZF) decoding have very low complexity, they suffer from unacceptable degradations in BLER performance. Sphere decoding (SD) is able to provide the BLER performance of ML detection with less complexity by searching only a subset of the entire lattice space. Nevertheless, it has been proven that its expected complexity is still exponential [1]. Thus it becomes impractical when the system order is high and the signal-to-noise ratio (SNR) is low.

The decoding algorithms based on semidefinite relaxation (SDR) approach have become more and more attractive simply because of the fact that semidefinite programming (SDP) problems can be efficiently solved in polynomial time [2-5]. The SDR approach was firstly applied to detect binary phase-shift keying (BPSK) and quadrature amplitude modulation (4-QAM) signals $[6,7]$. Then the extensions to different SDR techniques for 16-QAM signals had been proposed, such as polynomial-inspired SDR (PI-SDR) [8], boundconstrained SDR (BC-SDR) [9], and virtually antipodal SDR (VA-SDR) [10], all exhibiting acceptable BLER performance and relatively low complexity. In [11], it has been proved that there exists an equivalence among PI-SDR, BC-SDR, and VASDR for 16-QAM. The extension of BC-SDR and VA-SDR to 256-QAM has also been investigated, and both of them provide the same BLER performance. However, due to its high complexity, the PI-SDR is not suitable for extension to 256-QAM system.

In this paper, a new SDR detector is proposed for 256-QAM system [12]. Then, a comprehensive comparison between the proposed method and the previous SDR detectors is made. The results show that the proposed SDR can provide better BLER performance than both the BC-SDR and VA-SDR. Moreover, the complexity of the proposed 
method is higher than that of the BC-SDR but lower than that of the VA-SDR. This paper is organized as follows. In Section 2, the BC-SDR and VA-SDR are reviewed, and the proposed SDR is introduced. In Sections 3 and 4, the tightness and the complexity of these methods are analyzed. Then the simulation results are shown in Section 5. Finally, we conclude the paper in Section 6.

\section{Semidefinite Relaxation Detectors for 256-QAM MIMO System}

2.1. System Model. The system model for the MIMO transmission using $M$-QAM is considered in this paper. The MIMO system is modeled as

$$
\overline{\mathbf{r}}=\overline{\mathbf{H}} \overline{\mathbf{x}}+\overline{\mathbf{n}},
$$

where $\overline{\mathbf{r}}$ is the $N_{R}$-dimensional received signal vector and $\overline{\mathbf{x}}$ is the $N_{T}$-dimensional signal vector in the transmit lattice. $\overline{\mathbf{H}}$ denotes the channel matrix, with elements $h_{i j}$ representing the transfer function from the $j$ th transmit antenna to $i$ th receive antenna. $\overline{\mathbf{n}}$ is the $N_{R}$-dimensional additive noise. The signal vector $\overline{\mathbf{x}}$ is assumed to be a statistically independent variable with zero mean and variance $\sigma^{2}=N_{0} / 2$, where $N_{0}$ is noise power spectral density. Perfect channel knowledge is assumed to be known to the receiver. In addition, the channel matrix is assumed to be a flat fading channel and all its entries are complex Gaussian and independent. The noise is an independently and identically distributed (i.i.d.) zeromean Gaussian noise vector with elements having a fixed variance.

The complex transmission in (1) can be equivalently represented in real matrix form as

$$
\begin{gathered}
{\left[\begin{array}{c}
\operatorname{Re}(\overline{\mathbf{r}}) \\
\operatorname{Im}(\overline{\mathbf{r}})
\end{array}\right]=\left[\begin{array}{cc}
\operatorname{Re}(\overline{\mathbf{H}}) & -\operatorname{Im}(\overline{\mathbf{H}}) \\
\operatorname{Im}(\overline{\mathbf{H}}) & \operatorname{Re}(\overline{\mathbf{H}})
\end{array}\right]\left[\begin{array}{l}
\operatorname{Re}(\overline{\mathbf{x}}) \\
\operatorname{Im}(\overline{\mathbf{x}})
\end{array}\right]+\left[\begin{array}{c}
\operatorname{Re}(\overline{\mathbf{n}}) \\
\operatorname{Im}(\overline{\mathbf{n}})
\end{array}\right],} \\
\mathbf{r}=\mathbf{H} \mathbf{x}+\mathbf{n}
\end{gathered}
$$

with $\operatorname{Re}(\bullet)$ and $\operatorname{Im}(\bullet)$ being the real and imaginary parts of $(\bullet)$, respectively. The dimension of $\mathbf{x}$ and $\mathbf{r}$ is $M_{R}=$ $2 N_{R}$ and $M_{T}=2 N_{T}$, respectively. $\mathbf{H}$ becomes an $M_{R} \times$ $M_{T}$ matrix. And the noise $\mathbf{n}$ is an $M_{R}$-dimensional vector. ML decoding calculates the Euclidean distances between the possible transmit signal vectors and the received signal vector and then chooses the one with shortest distance as the solution. For MIMO system using 256-QAM, the ML detection aims at finding the solution of the following optimization problem:

$$
\begin{array}{ll}
\min & \|\mathbf{r}-\mathbf{H} \mathbf{x}\|^{2} \\
\text { st. } & \mathbf{x} \in\{ \pm 1, \pm 3, \pm 5, \pm 7, \pm 9, \pm 11, \pm 13, \pm 15\}^{M_{T}},
\end{array}
$$

where $\|\cdot\|$ represents the vector 2-norm. It is well known that ML detection can provide the best BLER performance. However, its computation requirement is too complicated to be implemented, especially for the cases with large number of antennas or high level modulation.
2.2. Review of $B C$-SDR. Define a rank-1 semidefinite matrix $\Omega$, which is given by

$$
\boldsymbol{\Omega}=\left[\begin{array}{ll}
\mathbf{x}^{T} & 1
\end{array}\right]^{T}\left[\begin{array}{ll}
\mathbf{x}^{T} & 1
\end{array}\right] .
$$

It is easy to find that the ML detection problem given in (3a) and (3b) can be rewritten as

$$
\begin{array}{ll}
\min & \operatorname{Tr}\left\{\boldsymbol{\Omega}\left[\begin{array}{cc}
\mathbf{H}^{T} \mathbf{H} & -\mathbf{H}^{T} \mathbf{r} \\
-\mathbf{r}^{T} \mathbf{H} & \mathbf{r}^{T} \mathbf{r}
\end{array}\right]\right\} \\
\text { st. } & \boldsymbol{\Omega}=\boldsymbol{\Omega}^{T} \in \mathbb{R}^{\left(M_{T}+1\right) \times\left(M_{T}+1\right)} \\
& \boldsymbol{\Omega}_{1,1}=\boldsymbol{\Omega}_{1,2} \boldsymbol{\Omega}_{2,1} \in \mathbb{R}^{M_{T} \times M_{T}} \\
& \boldsymbol{\Omega}_{1,2} \in\{ \pm 1, \pm 3, \pm 5, \pm 7, \pm 9, \pm 11, \pm 13, \pm 15\}^{M_{T}} \\
& \boldsymbol{\Omega}_{2,2}=1 .
\end{array}
$$

It can be observed that the high complexity of the ML detection is due to the presence of the two nonconvex constraints (5c) and (5d). Thus, relaxation of these constraints will be engaged to transform the original problem into a semidefinite problem, which can then be efficiently solved in polynomial time. First, constraint (5d) implies $1 \leq x_{i}^{2} \leq$ 225 , where $x_{i}$ denotes the $i$ th component of $\mathrm{x}$. Then, (5b) along with $(5 \mathrm{c})$ can be relaxed into $\mathbf{Y} \geq 0$. A new symbol $\mathbf{Y}$ is introduced here to distinguish $\mathbf{Y}$ from the aforementioned $\boldsymbol{\Omega}$ since they are actually different matrixes after relaxation. Consequently, the BC-SDR problem is obtained as

$$
\begin{array}{ll}
\min & \operatorname{Tr}\left\{\mathbf{Y}\left[\begin{array}{cc}
\mathbf{H}^{T} \mathbf{H} & -\mathbf{H}^{T} \mathbf{r} \\
-\mathbf{r}^{T} \mathbf{H} & \mathbf{r}^{T} \mathbf{r}
\end{array}\right]\right\} \\
\text { st. } & \mathbf{Y} \in \mathbb{R}^{\left(2 M_{T}+1\right) \times\left(2 M_{T}+1\right)} \succeq 0 \\
& \mathbf{I} \preceq \operatorname{Diag}\left\{\mathbf{Y}_{1,1}\right\} \preceq 255 \mathbf{I}, \quad \mathbf{I} \in \mathbb{R}^{M_{T} \times M_{T}} \\
& \mathbf{Y}_{2,2}=1 .
\end{array}
$$

The BC-SDR problem (6a), (6b), (6c), and (6d) can then be solved by any of the SDP solvers, such as Sedumi [3], based on interior point methods. Although (6b) could be deduced from (5b) and (5c) and (6c) can also be deduced from (5d), problem (6a), (6b), (6c), and (6d) is however not exactly equivalent to problem $(5 a),(5 b),(5 c),(5 d)$, and (5e). Thus, the solution obtained by solving (6a), (6b), (6c), and (6d) has more errors than ML solution in (5a), (5b), (5c), (5d), and (5e). Once the solution is found, the randomization approach is applied to quantize the resulting $\widehat{\mathbf{Y}}_{1,2}$ till constraint (3b) is satisfied.

2.3. Review of VA-SDR. It is worth noting that when constraint (3b) is expected to be satisfied, the signal $\mathbf{x}$ could be expressed as

$$
\mathbf{x}=\mathbf{U} \mathbf{p}^{T}
$$

where $\mathbf{U}=\left[\begin{array}{llll}\mathbf{I} & 2 \mathbf{I} & 4 \mathbf{I} & 8 \mathbf{I}\end{array}\right], \mathbf{p}=\left[\begin{array}{llll}\mathbf{p}_{1} & \mathbf{p}_{2} & \mathbf{p}_{3} & \mathbf{p}_{4}\end{array}\right], \mathbf{I} \in \mathbb{R}^{M_{T} \times M_{T}}$, and $\mathbf{p}_{i} \in\{ \pm 1\}^{M_{T}}, i=1,2,3,4$. 
TABLE 1: The value of $\mathbf{x}$.

\begin{tabular}{lccccc}
\hline $\mathbf{q}_{2}$ & & & $\mathbf{q}_{1}$ & & \\
\hline-3 & -3 & -1 & -7 & 1 & 3 \\
-1 & -15 & -5 & 3 & 11 \\
1 & -13 & -3 & 5 & 13 \\
3 & -11 & -1 & 7 & 15 \\
\hline
\end{tabular}

Substituting (7) into (4) and defining a matrix $\mathbf{Z}$, which is given by

$$
\mathbf{Z}=\left[\begin{array}{ll}
\mathbf{p} & 1
\end{array}\right]^{T}\left[\begin{array}{ll}
\mathbf{p} & 1
\end{array}\right]
$$

the objective function (5a) can be equivalently transformed into (9a), and constraints (5d) and (5e) are equal to (9b). Similarly, (5b) along with (5c) can be relaxed into (9c). Thus, we obtain the VA-SDR problem given by

$$
\begin{array}{ll}
\min & \operatorname{Tr}\left\{\mathbf{Z}\left[\begin{array}{cc}
\mathbf{U}^{T} \mathbf{H}^{T} \mathbf{H U} & -\mathbf{U}^{T} \mathbf{H}^{T} \mathbf{r} \\
-\mathbf{r}^{T} \mathbf{H} \mathbf{U} & \mathbf{r}^{T} \mathbf{r}
\end{array}\right]\right\} \\
\text { st. } & \mathbf{Z} \in \mathbb{R}^{\left(4 M_{T}+1\right) \times\left(4 M_{T}+1\right)} \geq 0 \\
& \operatorname{Diag}(\mathbf{Z})=1 \mathbf{I} .
\end{array}
$$

Since the first $4 M_{T}$ elements of the last row in the solution $\widehat{\mathbf{Z}}$ can be considered as $\widehat{\mathbf{p}}=\left[\begin{array}{llll}\widehat{\mathbf{p}}_{1} & \widehat{\mathbf{p}}_{2} & \widehat{\mathbf{p}}_{3} & \widehat{\mathbf{p}}_{4}\end{array}\right]$, thus the optimum solution $\widehat{\mathbf{x}}$ is reconstructed by using (7). Finally, the solution is quantized by using randomization.

2.4. Proposed SDR. Considering constraint (3b), the signal $\mathbf{x}$ could also be expressed as

$$
\mathbf{x}=\mathbf{V} \mathbf{q}^{T}
$$

where $\mathbf{V}=\left[\begin{array}{ll}\mathbf{I} & 4 \mathbf{I}\end{array}\right], \mathbf{q}=\left[\begin{array}{ll}\mathbf{q}_{1} & \mathbf{q}_{2}\end{array}\right], \mathbf{I} \in \mathbb{R}^{M_{T} \times M_{T}}$, and $\mathbf{q}_{1}, \mathbf{q}_{2} \in$ $\{ \pm 1, \pm 3\}^{M_{T}}$.

Table 1 gives the value of $x_{j}$ for the possible combinations of $q_{1 j}$ and $q_{2 j}$, where $x_{j}, q_{1 j}$, and $q_{2 j}$ denote the $j$ th element of $\mathbf{x}, \mathbf{q}_{1}$, and $\mathbf{q}_{2}$, respectively.

By substituting (10) into (4) and defining a matrix W, which is given by

$$
\mathbf{W}=\left[\begin{array}{ll}
\mathbf{q} & 1
\end{array}\right]^{T}\left[\begin{array}{ll}
\mathbf{q} & 1
\end{array}\right]
$$

the objective function (5a) can be equivalently transformed into (12a). Moreover, it can be known from (10) that constraint (5d) is equivalent to $\mathbf{q}_{1}, \mathbf{q}_{2} \in\{ \pm 1, \pm 3\}^{M_{T}}$, which are essentially the well-known indices used to characterize the 16QAM constellation. Herein, the set operation method [13] is engaged to formulate the alphabet constraint (5d) into (12b) and (12c). Similarly, (5b) along with (5c) can be relaxed into (12d). Also (5e) can be reformulated as (12e). Thus, we obtain the proposed SDR problem given by

$$
\begin{array}{ll}
\min & \operatorname{Tr}\left\{\mathbf{W}\left[\begin{array}{cc}
\mathbf{V}^{T} \mathbf{H}^{T} \mathbf{H V} & -\mathbf{V}^{T} \mathbf{H}^{T} \mathbf{r} \\
-\mathbf{r}^{T} \mathbf{H} \mathbf{V} & \mathbf{r}^{T} \mathbf{r}
\end{array}\right]\right\} \\
\text { st. } & 1 \mathbf{I} \preceq \operatorname{Diag}\left\{\mathbf{W}_{1,1}\right\} \preceq 9 \mathbf{I}, \quad \mathbf{I} \in \mathbb{R}^{2 M_{T} \times 2 M_{T}}, \\
& \operatorname{Diag}\left\{\mathbf{W}_{1,1}\right\} \pm 4 \operatorname{Diag}\left\{\mathbf{W}_{1,2}\right\}+3 \mathbf{I} \succeq 0, \quad \mathbf{I} \in \mathbb{R}^{2 M_{T} \times 2 M_{T}}, \\
& \mathbf{W} \in \mathbb{R}^{\left(2 M_{T}+1\right) \times\left(2 M_{T}+1\right)} \geq 0, \\
& \mathbf{W}_{2,2}=1 .
\end{array}
$$

Similar to the VA-SDR method, the first $2 M_{T}$ elements of the last row in the solution $\widehat{\mathbf{W}}$ can be considered as $\widehat{\mathbf{q}}=\left[\begin{array}{ll}\widehat{\mathbf{q}}_{1} & \widehat{\mathbf{q}}_{2}\end{array}\right]$; thus, the optimum solution $\widehat{\mathbf{x}}$ is reconstructed by using (10). Finally, the solution is quantized by using randomization.

\section{Comparison of Tightness}

As mentioned in Section 2, these three SDR problems are all relaxed from the original problem (3a) and (3b), and their objective functions are equivalent, which are actually calculating the Euclidean distance given by (3a). Thus, the tightness of the constraints of each SDR algorithm implies how close it is to the ML decoding. In what follows, we will compare their tightness.

3.1. Equivalence of $B C-S D R$ and VA-SDR. Firstly, we will demonstrate that the constraints of the BC-SDR problem are equivalent to those of the VA-SDR problem. This equivalence has been considered in [11], and for completeness of understanding, we will reorganize the proof in the following two steps.

Step 1. For each matrix $\mathbf{Z} \in \mathbb{R}^{\left(4 M_{T}+1\right) \times\left(4 M_{T}+1\right)}$ that satisfies constraints (9b) and (9c), there must be a matrix $Y \in$ $\mathbb{R}^{\left(M_{T}+1\right) \times\left(M_{T}+1\right)}$ which satisfies constraints $(6 \mathrm{~b})-(6 \mathrm{~d})$.

Proof. For any matrix $\mathbf{Z}$ which satisfies constraints (9b) and (9c), it has the following form:

$$
\mathbf{Z}=\left[\begin{array}{cc}
\mathbf{Z}_{1,1} \in \mathbb{R}^{4 M_{T} \times 4 M_{T}} & \mathbf{Z}_{1,2} \in \mathbb{R}^{4 M_{T} \times 1} \\
\mathbf{Z}_{2,1} \in \mathbb{R}^{1 \times 4 M_{T}} & 1
\end{array}\right]
$$

Since $\mathbf{Z} \geq 0$, there should be a reversible matrix:

$$
\begin{gathered}
\Lambda=\left[\lambda_{1}, \lambda_{2}, \ldots, \lambda_{M_{t}}, \lambda_{M_{t}+1}, \lambda_{M_{t}+2}, \ldots, \lambda_{2 M_{t}}, \lambda_{2 M_{t}+1}, \lambda_{2 M_{t}+2}\right. \\
\left.\ldots \lambda_{3 M_{t}}, \lambda_{3 M_{t}+1}, \lambda_{3 M_{t}+2}, \ldots, \lambda_{4 M_{t}}, \lambda_{4 M_{t}+1}\right]
\end{gathered}
$$


that satisfies

$$
\mathbf{Z}=\boldsymbol{\Lambda}^{T} \boldsymbol{\Lambda}
$$

where $\lambda_{m} \in \mathbb{R}^{\left(4 M_{T}+1\right) \times 1}, m=1,2, \ldots, 4 M_{T}+1$.

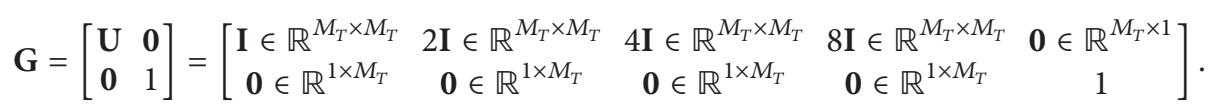

From (13)-(15) and (9c), we get

$$
\begin{aligned}
\left\|\boldsymbol{\lambda}_{n}\right\|=\left(\boldsymbol{\lambda}_{n}^{T} \boldsymbol{\lambda}_{n}\right)^{1 / 2}=1, \quad n=1,2, \ldots, 4 M_{T}, \quad \text { (18) } & \text { Substituting (14) into (16) gives } \\
\mathbf{Y} & =\left[\begin{array}{ll}
\mathbf{Y}_{1,1} & \mathbf{Y}_{1,2} \\
\mathbf{Y}_{2,1} & \mathbf{Y}_{2,2}
\end{array}\right] \\
& =\left[\begin{array}{cc}
\left(\boldsymbol{\Lambda}_{1}+2 \boldsymbol{\Lambda}_{2}+4 \boldsymbol{\Lambda}_{3}+8 \boldsymbol{\Lambda}_{4}\right)^{T}\left(\boldsymbol{\Lambda}_{1}+2 \boldsymbol{\Lambda}_{2}+4 \boldsymbol{\Lambda}_{3}+8 \boldsymbol{\Lambda}_{4}\right) & \left(\boldsymbol{\Lambda}_{1}+2 \boldsymbol{\Lambda}_{2}+4 \boldsymbol{\Lambda}_{3}+8 \boldsymbol{\Lambda}_{4}\right)^{T} \boldsymbol{\lambda}_{4 M_{T}+1} \\
\boldsymbol{\lambda}_{4 M_{T}+1}^{T}\left(\boldsymbol{\Lambda}_{1}+2 \boldsymbol{\Lambda}_{2}+4 \boldsymbol{\Lambda}_{3}+8 \boldsymbol{\Lambda}_{4}\right)^{T} & \boldsymbol{\lambda}_{4 M_{T}+1}^{T} \boldsymbol{\lambda}_{4 M_{T}+1}
\end{array}\right]
\end{aligned}
$$

where $\Lambda_{1}=\left[\lambda_{1}, \lambda_{2}, \ldots, \lambda_{N_{t}}\right] \in \mathbb{R}^{\left(4 M_{T}+1\right) \times M_{T}}, \Lambda_{2}=\left[\lambda_{M_{T}+1}\right.$, $\left.\lambda_{M_{T}+2}, \ldots, \lambda_{2 M_{T}}\right] \in \mathbb{R}^{\left(4 M_{T}+1\right) \times M_{T}}, \Lambda_{3}=\left[\lambda_{2 N_{t}+1}, \lambda_{2 N_{t}+2}, \ldots\right.$, $\left.\lambda_{3 N_{t}}\right] \in \mathbb{R}^{\left(4 M_{T}+1\right) \times M_{T}}$, and $\Lambda_{4}=\left[\lambda_{3 N_{t}+1}, \lambda_{3 N_{t}+2}, \ldots, \lambda_{4 N_{t}}\right] \in$ $\mathbb{R}^{\left(4 M_{T}+1\right) \times M_{T}}$.

From (19) and (20) it can be known that $\mathbf{Y}_{2,2}=1$, and ( $\left.6 \mathrm{~d}\right)$ is satisfied. Moreover, we have

$$
\mathbf{Y}_{1,1}=\left(\Lambda_{1}+2 \Lambda_{2}+4 \Lambda_{3}+8 \Lambda_{4}\right)^{T}\left(\Lambda_{1}+2 \Lambda_{2}+4 \Lambda_{3}+8 \Lambda_{4}\right) .
$$

The element located in the $j$ th row and the $j$ th column of $\mathbf{Y}_{1,1}$ is

$$
y_{j j}=\left\|\lambda_{j}+2 \lambda_{M_{T}+j}+4 \lambda_{2 M_{T}+j}+8 \lambda_{3 M_{T}+j}\right\|^{2} .
$$

From the perspective of geometry and (18), it is easy to know that

$$
\begin{aligned}
1 & =\left\|8 \lambda_{3 M_{T}+j}\right\|-\left\|\lambda_{j}\right\|-\left\|2 \lambda_{M_{T}+j}\right\|-\left\|4 \lambda_{2 M_{T}+j}\right\| \\
& \leq\left\|\lambda_{j}+2 \lambda_{M_{T}+j}+4 \lambda_{2 M_{T}+j}+8 \lambda_{3 M_{T}+j}\right\| \\
& \leq\left\|\lambda_{j}\right\|+\left\|2 \lambda_{M_{T}+j}\right\|+\left\|4 \lambda_{2 M_{T}+j}\right\|+\left\|8 \lambda_{3 M_{T}+j}\right\|=15 .
\end{aligned}
$$

Thus we have

$$
\begin{gathered}
1 \leq y_{j j} \leq 225, \\
1 \mathbf{I} \preceq \operatorname{Diag}\left\{\mathbf{Y}_{1,1}\right\} \preceq 225 \mathrm{I} .
\end{gathered}
$$

The proof is complete.

Step 2. For each matrix $\mathbf{Y} \in \mathbb{R}^{\left(M_{T}+1\right) \times\left(M_{T}+1\right)}$ that satisfies constraints (6b)-(6d), there must be a matrix $\mathbf{Z} \in$ $\mathbb{R}^{\left(4 M_{T}+1\right) \times\left(4 M_{T}+1\right)}$ which satisfies constraints (9b) and (9c).
Proof. For any matrix $\mathbf{Y}$ that satisfies constraints (6b)-(6d), it has the following form:

$$
\mathbf{Y}=\left[\begin{array}{cc}
\mathbf{Y}_{1,1} \in \mathbb{R}^{M_{T} \times M_{T}} & \mathbf{Y}_{1,2} \in \mathbb{R}^{M_{T} \times 1} \\
\mathbf{Y}_{2,1} \in \mathbb{R}^{1 \times M_{T}} & 1
\end{array}\right]
$$

Since $\mathbf{Y} \geq 0$, there must be a reversible matrix $\Gamma=\left[\gamma_{1}, \gamma_{2}\right.$, $\left.\gamma_{3}, \ldots, \bar{\gamma}_{M_{T}}, \gamma_{M_{T}+1}\right]$ that satisfies

$$
\mathbf{Y}=\Gamma^{T} \Gamma
$$

where $\boldsymbol{\gamma}_{l} \in \mathbb{R}^{\left(M_{T}+1\right) \times 1}, l=1,2, \ldots, M_{T}+1$.

From (25) and (26) and (6c), it can be obtained that

$$
\begin{gathered}
\left\|\gamma_{M_{T}+1}\right\|=\left(\gamma_{M_{T}+1}^{T} \gamma_{M_{T}+1}\right)^{1 / 2}=1, \\
1 \leq\left\|\gamma_{j}\right\|=\left(\gamma_{j}^{T} \gamma_{j}\right)^{1 / 2} \leq 15, \quad j=1,2, \ldots, M_{T} .
\end{gathered}
$$

From the perspective of geometry, it is easy to know that there should be vectors $\gamma_{j 1}, \gamma_{j 2}, \gamma_{j 3}, \gamma_{j 4} \in \mathbb{R}^{\left(M_{T}+1\right) \times 1}$, which satisfy

$$
\begin{gathered}
\gamma_{j}=\gamma_{j 1}+2 \gamma_{j 2}+4 \gamma_{j 3}+8 \gamma_{j 4}, \\
\left\|\gamma_{j 1}\right\|=\left\|\gamma_{j 2}\right\|=\left\|\gamma_{j 3}\right\|=\left\|\gamma_{j 4}\right\|=1 .
\end{gathered}
$$

Now we construct another matrix on the basis of (26) and (28), which is given by

$$
\begin{aligned}
& \Gamma^{\prime}= {\left[\gamma_{11}, \gamma_{12}, \gamma_{13}, \gamma_{14}, \gamma_{21}, \gamma_{22}, \gamma_{23}, \gamma_{24}, \ldots, \gamma_{M_{t} 1},\right.} \\
&\left.\gamma_{M_{t} 2}, \gamma_{M_{t} 3}, \gamma_{M_{t} 4}, \gamma_{M_{t}+1}\right] \\
& \in \mathbb{R}^{\left(M_{T}+1\right) \times\left(4 M_{T}+1\right)} .
\end{aligned}
$$


Next, we construct a semidefinite matrix $\mathbf{Z} \in$ $\mathbb{R}^{\left(4 M_{T}+1\right) \times\left(4 M_{T}+1\right)}$, which is defined as

$$
\mathbf{Z}=\left(\boldsymbol{\Gamma}^{\prime}\right)^{T} \boldsymbol{\Gamma}^{\prime} .
$$

Substituting (30) into (31), the element located in the $(j \times i)$ th row and the $(j \times i)$ th column of $\mathbf{Z}$ is given as

$$
z_{(j i)(j i)}=\left(\gamma_{j i}^{T} \gamma_{j i}\right)=\left\|\gamma_{j i}\right\|^{2}=1,
$$

where $j=1,2, \ldots, M_{T}, i=1,2,3,4$. Moreover, the $\left(4 M_{T}+\right.$ 1)th row and the $\left(4 M_{T}+1\right)$ th column of $\mathbf{Z}$ are

$$
z_{\left(4 M_{T}+1\right)\left(4 M_{T}+1\right)}=\left(\gamma_{M_{T}+1}^{T} \gamma_{M_{T}+1}\right)=1 .
$$

From (32) and (33), it can be obtained that

$$
\operatorname{Diag}(\mathbf{Z})=1 \mathbf{I} .
$$

The proof is complete.

From both Steps 1 and 2, the equivalence of BC-SDR and VA-SDR can be concluded.

3.2. Proposed SDR Tighter Than BC-SDR and VA-SDR. Secondly, we will demonstrate that the constraints of proposed SDR problem are tighter than those of the BC-SDR problem and also tighter than those of the VA-SDR due to the aforementioned equivalence. For this purpose, a new SDR problem is constructed given by

$$
\begin{array}{ll}
\min & \operatorname{Tr}\left\{\mathbf{W}^{\prime}\left[\begin{array}{cc}
\mathbf{V}^{T} \mathbf{H}^{T} \mathbf{H V} & -\mathbf{V}^{T} \mathbf{H}^{T} \mathbf{r} \\
-\mathbf{r}^{T} \mathbf{H} \mathbf{V} & \mathbf{r}^{T} \mathbf{r}
\end{array}\right]\right\} \\
\text { st. } & 1 \mathbf{I} \preceq \operatorname{Diag}\left\{\mathbf{W}_{1,1}^{\prime}\right\} \preceq 9 \mathbf{I}, \quad \mathbf{I} \in \mathbb{R}^{2 M_{T} \times 2 M_{T}}, \\
& \mathbf{W}^{\prime} \in \mathbb{R}^{\left(2 M_{T}+1\right) \times\left(2 M_{T}+1\right)} \succeq 0, \\
& \mathbf{W}_{2,2}^{\prime}=1 .
\end{array}
$$

By comparing (35a), (35b), (35c), and (35d) and (12a), (12b), (12c), (12d), and (12e), it is apparent that the proposed SDR is tighter than the new SDR since the proposed SDR is nearly the same as the new SDR except that the proposed SDR has an extra constraint (12c). In what follows, we will prove that the new SDR given by (35a), (35b), (35c), and (35d) is equivalent to BC-SDR given by (6a), (6b), (6c), and (6d), and therefore the proposed SDR being tighter than BC-SDR can be proven.

Step 1. For each matrix $\mathbf{W}^{\prime} \in \mathbb{R}^{\left(2 M_{T}+1\right) \times\left(2 M_{T}+1\right)}$ that satisfies constraints (35b)-(35d), there must be a matrix $\mathbf{Y} \in$ $\mathbb{R}^{\left(M_{T}+1\right) \times\left(M_{T}+1\right)}$ which satisfies constraints $(6 \mathrm{~b})-(6 \mathrm{~d})$.

Proof. For any matrix $\mathbf{W}^{\prime}$ that satisfies constraints (35b)(35d), it has the following form:

$$
\mathbf{W}^{\prime}=\left[\begin{array}{cc}
\mathbf{W}_{1,1}^{\prime} \in \mathbb{R}^{2 M_{T} \times 2 M_{T}} & \mathbf{W}_{1,2}^{\prime} \in \mathbb{R}^{2 M_{T} \times 1} \\
\mathbf{W}_{2,1}^{\prime} \in \mathbb{R}^{1 \times 2 M_{T}} & 1
\end{array}\right] .
$$

Since $\mathbf{W}^{\prime} \succ 0$, there should be a reversible matrix:

$$
\begin{aligned}
& \Psi \\
& =\left[\psi_{1}, \psi_{2}, \ldots, \psi_{M_{T}}, \psi_{M_{T}+1}, \psi_{M_{T}+2}, \ldots, \psi_{2 M_{T}}, \psi_{2 M_{T}+1}\right]
\end{aligned}
$$

that satisfies

$$
\mathbf{W}^{\prime}=\Psi^{T} \Psi
$$

where $\psi_{s} \in \mathbb{R}^{\left(2 M_{T}+1\right) \times 1}, s=1,2, \ldots, 2 M_{T}+1$.

From (36)-(38), (35b) and (35d) give

$$
\begin{gathered}
1 \leq\left\|\psi_{k}\right\|=\left(\psi_{k}^{T} \psi_{k}\right)^{1 / 2} \leq 3, \quad k=1,2, \ldots, 2 M_{T}, \\
\psi_{2 M_{T}+1}^{T} \psi_{2 M_{T}+1}=1 .
\end{gathered}
$$

Now we construct a semidefinite matrix $\mathbf{Y} \in \mathbb{R}^{\left(M_{T}+1\right) \times\left(M_{T}+1\right)}$ which is defined as

$$
\mathbf{Y}=\mathbf{E W}^{\prime} \mathbf{E}^{T}=\left(\Psi \mathbf{E}^{T}\right)^{T}\left(\Psi \mathbf{E}^{T}\right),
$$

where

$$
\begin{aligned}
\mathbf{E} & =\left[\begin{array}{ll}
\mathbf{V} & 0 \\
\mathbf{0} & 1
\end{array}\right] \\
& =\left[\begin{array}{ccc}
\mathbf{I} \in \mathbb{R}^{M_{T} \times M_{T}} & 4 \mathbf{I} \in \mathbb{R}^{M_{T} \times M_{T}} & \mathbf{0} \in \mathbb{R}^{M_{T} \times 1} \\
\mathbf{0} \in \mathbb{R}^{1 \times M_{T}} & \mathbf{0} \in \mathbb{R}^{1 \times M_{T}} & 1
\end{array}\right] .
\end{aligned}
$$

Substituting (38) into (41) gives

$$
\begin{aligned}
\mathbf{Y} & =\left[\begin{array}{ll}
\mathbf{Y}_{1,1} & \mathbf{Y}_{1,2} \\
\mathbf{Y}_{2,1} & \mathbf{Y}_{2,2}
\end{array}\right] \\
& =\left[\begin{array}{cc}
\left(\boldsymbol{\Psi}_{1}+4 \boldsymbol{\Psi}_{2}\right)^{T}\left(\boldsymbol{\Psi}_{1}+4 \boldsymbol{\Psi}_{2}\right) & \left(\boldsymbol{\Psi}_{1}+4 \boldsymbol{\Psi}_{2}\right)^{T} \boldsymbol{\psi}_{2 M_{T}+1} \\
\boldsymbol{\psi}_{2 M_{T}+1}^{T}\left(\boldsymbol{\Psi}_{1}+4 \boldsymbol{\Psi}_{2}\right)^{T} & \boldsymbol{\psi}_{2 M_{T}+1}^{T} \boldsymbol{\psi}_{2 M_{T}+1}
\end{array}\right],
\end{aligned}
$$

where

$$
\begin{gathered}
\Psi_{1}=\left[\psi_{1}, \psi_{2}, \ldots, \psi_{N_{t}}\right] \in \mathbb{R}^{\left(2 M_{T}+1\right) \times M_{T}}, \\
\Psi_{2}=\left[\psi_{M_{T}+1}, \psi_{M_{T}+2}, \ldots, \psi_{2 M_{T}}\right] \in \mathbb{R}^{\left(2 M_{T}+1\right) \times M_{T}} .
\end{gathered}
$$

From (40) and (43) it can be known that $\mathbf{Y}_{2,2}=1$, and (6d) is satisfied. Moreover, we have

$$
\mathbf{Y}_{1,1}=\left(\Psi_{1}+4 \Psi_{2}\right)^{T}\left(\Psi_{1}+4 \Psi_{2}\right)
$$

and the element located in the $j$ th row and the $j$ th column of $\mathbf{Y}_{1,1}$ is

$$
y_{j j}=\left\|\psi_{j}+4 \psi_{N_{t}+j}\right\|^{2}, \quad j=1,2, \ldots, M_{T} .
$$

From the perspective of geometry and (39), it is easy to know that

$$
\begin{aligned}
1 & \leq\left\|4 \psi_{M_{T}+j}\right\|-\left\|\psi_{j}\right\| \leq\left\|\psi_{j}+4 \psi_{M_{T}+j}\right\| \\
& \leq\left\|\psi_{j}\right\|+\left\|4 \psi_{M_{T}+j}\right\| \leq 15 .
\end{aligned}
$$


Thus we have

$$
\begin{gathered}
1 \leq y_{j j} \leq 225, \\
1 \mathbf{I} \preceq \operatorname{Diag}\left\{\mathbf{Y}_{1,1}\right\} \preceq 225 \mathbf{I} .
\end{gathered}
$$

The proof is complete.

Step 2. For each matrix $\mathbf{Y} \in \mathbb{R}^{\left(M_{T}+1\right) \times\left(M_{T}+1\right)}$ that satisfies constraints $(6 \mathrm{~b})-(6 \mathrm{~d})$, there must be a matrix $\mathbf{W}^{\prime} \in$ $\mathbb{R}^{\left(2 M_{T}+1\right) \times\left(2 M_{T}+1\right)}$ which satisfies constraints (35b)-(35d).

Proof. For any matrix $\mathbf{Y}$ that satisfies constraints (6b)-(6d), it has the following form:

$$
\mathbf{Y}=\left[\begin{array}{cc}
\mathbf{Y}_{1,1} \in \mathbb{R}^{M_{T} \times M_{T}} & \mathbf{Y}_{1,2} \in \mathbb{R}^{M_{T} \times 1} \\
\mathbf{Y}_{2,1} \in \mathbb{R}^{1 \times M_{T}} & 1
\end{array}\right]
$$

Since $\mathrm{Y} \succ 0$, there must be a reversible matrix $\Delta=$ $\left[\boldsymbol{\sigma}_{1}, \boldsymbol{\sigma}_{2}, \overline{\sigma_{3}}, \ldots, \sigma_{M_{T}}, \sigma_{M_{T}+1}\right]$ that satisfies

$$
\mathbf{Y}=\boldsymbol{\Delta}^{T} \boldsymbol{\Delta}
$$

where $\sigma_{l} \in \mathbb{R}^{\left(M_{T}+1\right) \times 1}, l=1,2, \ldots, M_{T}+1$.

From (49), (50), and (6c), it can be obtained that

$$
\begin{gathered}
\left\|\boldsymbol{\sigma}_{M_{T}+1}\right\|=\left(\boldsymbol{\sigma}_{M_{T}+1}^{T} \boldsymbol{\sigma}_{M_{T}+1}\right)^{1 / 2}=1, \\
1 \leq\left\|\boldsymbol{\sigma}_{j}\right\|=\left(\boldsymbol{\sigma}_{j}^{T} \boldsymbol{\sigma}_{j}\right)^{1 / 2} \leq 15, \quad j=1,2, \ldots, M_{T} .
\end{gathered}
$$

From the perspective of geometry, it is easy to know that there should be vectors $\boldsymbol{\sigma}_{j 1}, \boldsymbol{\sigma}_{j 2} \in \mathbb{R}^{\left(M_{T}+1\right) \times 1}$, which satisfy

$$
\begin{gathered}
\boldsymbol{\sigma}_{j}=\boldsymbol{\sigma}_{j 1}+4 \boldsymbol{\sigma}_{j 2}, \\
1 \leq\left\|\boldsymbol{\sigma}_{j i}\right\|=\left(\boldsymbol{\sigma}_{j i}^{T} \boldsymbol{\sigma}_{j i}\right)^{1 / 2} \leq 3, \quad i=1,2 .
\end{gathered}
$$

Now we construct another matrix on the basis of (50) and (52), which is given by

$$
\begin{aligned}
\Delta^{\prime} & =\left[\boldsymbol{\sigma}_{11}, \boldsymbol{\sigma}_{12}, \boldsymbol{\sigma}_{21}, \boldsymbol{\sigma}_{22}, \ldots, \boldsymbol{\sigma}_{M_{t} 1}, \boldsymbol{\sigma}_{M_{t} 2}, \boldsymbol{\sigma}_{M_{t}+1}\right] \\
& \in \mathbb{R}^{\left(M_{T}+1\right) \times\left(2 M_{T}+1\right)} .
\end{aligned}
$$

Next, we construct a semidefinite matrix $\mathbf{W}^{\prime} \in$ $\mathbb{R}^{\left(2 M_{T}+1\right) \times\left(2 M_{T}+1\right)}$ which is defined as

$$
\mathbf{W}^{\prime}=\left(\Delta^{\prime}\right)^{T} \Delta^{\prime} \text {. }
$$

Substituting (54) into (55), the element located in the $(j \times i)$ th row and the $(j \times i)$ th column of $\mathbf{W}^{\prime}$ is given as

$$
w_{(j i)(j i)}^{\prime}=\left(\boldsymbol{\sigma}_{j i}^{T} \boldsymbol{\sigma}_{j i}\right)=\left\|\boldsymbol{\sigma}_{j i}\right\|^{2},
$$

where $j=1,2, \ldots, M_{T}, i=1,2$.

Then, from (53) and (56) it can be obtained that

$$
1 \mathbf{I} \preceq \operatorname{Diag}\left\{\mathbf{W}_{1,1}^{\prime}\right\} \preceq 9 \mathbf{I}, \quad \mathbf{I} \in \mathbb{R}^{2 M_{T} \times 2 M_{T}} .
$$

Moreover, the $\left(2 M_{T}+1\right)$ th row and the $\left(2 M_{T}+1\right)$ th column of $\mathbf{W}^{\prime}$ are

$$
\mathbf{W}_{2,2}^{\prime}=w_{\left(2 M_{T}+1\right)\left(2 M_{T}+1\right)}^{\prime}=\left(\boldsymbol{\sigma}_{M_{T}+1}^{T} \boldsymbol{\sigma}_{M_{T}+1}\right)=1 .
$$

The proof is complete.
From both Steps 1 and 2, the equivalence of BC-SDR and new SDR given by (35a), (35b), (35c), and (35d) can be concluded. Therefore, the constraints of the proposed SDR are tighter than those of the BC-SDR and also tighter than those of the VA-SDR due to the aforementioned equivalence.

\section{Comparison of Complexity}

Firstly, the BC-SDR given in (6a) $-(6 \mathrm{~d})$ consists of a $\left(M_{T}+1\right) \times$ $\left(M_{T}+1\right)$ matrix variable $\mathbf{Y}$ and $O\left(M_{T}+1\right)$ linear constraints. Since constraints $(6 \mathrm{c})$ and $(6 \mathrm{~d})$ are separable, the complexity of the BC-SDR detector is $O\left(\left(M_{T}+1\right)^{3.5}\right)$ [9]. Secondly, the VA-SDR given in (9a)-(9c) involves a $\left(4 M_{T}+1\right) \times\left(4 M_{T}+1\right)$ matrix, variable $\mathbf{Z}$, and $O\left(4 M_{T}+1\right)$. The constraint $(9 \mathrm{c})$ is also separable; thus, the complexity of the VA-SDR detector is $O\left(\left(4 M_{T}+1\right)^{3.5}\right)$. Finally, the proposed SDR shown in (12a)(12e) consists of a $\left(2 M_{T}+1\right) \times\left(2 M_{T}+1\right)$ matrix, variable $\mathbf{W}$, and two $O\left(4 M_{T}\right)$ linear constraints. According to $[13,14]$, it can be known that the complexity of the proposed SDR detector is $O\left(4\left(2 M_{T}+1\right)^{4.5}+8\left(2 M_{T}+1\right)^{3.5}\right)$.

\section{Simulation Results}

Computer simulations were conducted to evaluate the performance of these three SDR detectors. An uncoded MIMO system with independent Rayleigh fading channel was taken into account and the Sedumi toolbox within Matlab software was used to implement the SDR detection algorithms. Figures 1 and 2 show the BLER performances of the three SDR detectors for $4 \times 4$ and $8 \times 8256$-QAM systems, respectively. It can be observed that the BC-SDR detector and the VASDR detector provide exactly the same BLER performance, while the proposed SDR detector can provide the best BLER performance among these three detectors. This agrees well with the analysis presented in Section 3. What is more, it can be found that the improvement concerning the BLER performance provided by the proposed SDR detector in the case of $8 \times 8 \mathrm{MIMO}$ system is larger than that in the case of $4 \times 4 \mathrm{MIMO}$ system. The relaxation of the alphabet set engaged in the BC-SDR and the VA-SDR will cause increasing errors with the increase of the number of the antennas. That is because the inaccurate detection of any dimension of the transmitted signal will result in the failure of the detection of the whole signal vector, and the higher the dimension of the problem is, the bigger the possibility of failure will be. The main merit of the proposed SDR over the BC-SDR and the VA-SDR is that it can offer more accurate relaxation of the alphabet set. Moreover, the computational times for solving one signal vector of these SDR detectors are illustrated in Figures 3 and 4 . These figures demonstrate that the BC-SDR has the lowest computational complexity and the VA-SDR has the highest computational complexity while the proposed SDR is in between the two detection methods. It is also found that the complexity of SDR detectors is independent of SNR. This is a distinct advantage over the SD whose complexity varies as a function of SNR, making SD difficult to be implemented in practice. 


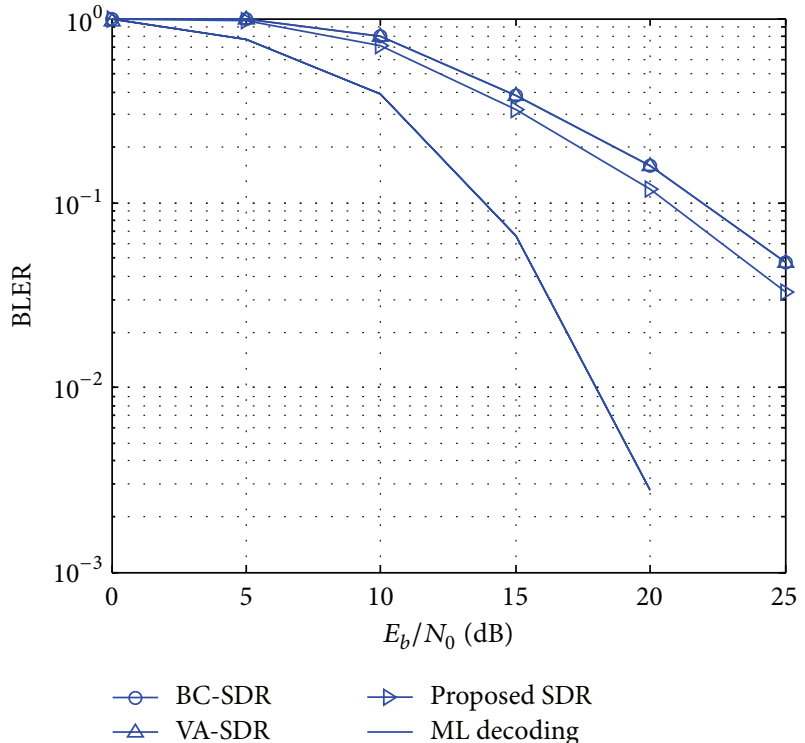

FIGURE 1: The BLER performance of the SDR detectors for $4 \times 4256$ QAM MIMO systems.

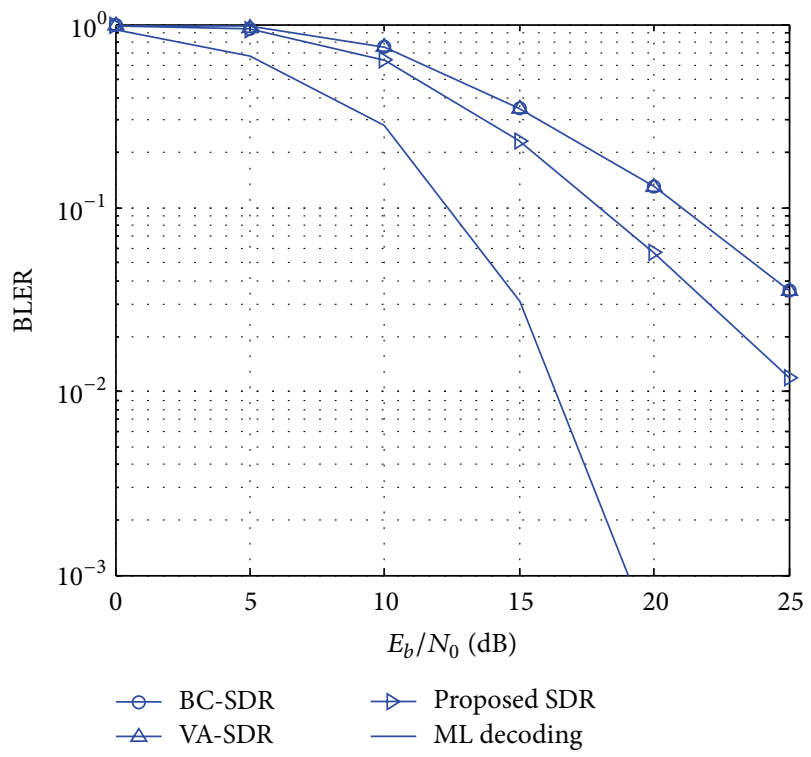

FIGURE 2: The BLER performance of the SDR detectors for $8 \times 8$ 256-QAM MIMO systems.

\section{Conclusion}

In this paper, we proposed a SDR detector for 256-QAM MIMO system and also reviewed two other SDR detectors, namely, BC-SDR detector and VA-SDR detector. Then we analyzed the tightness and the complexity of these three SDR detectors. Both theoretical analysis and simulation results demonstrate that the proposed SDR can provide the best BLER performance among these three detectors, while the BC-SDR detector and the VA-SDR detector provide exactly the same BLER performance. Moreover, the BC-SDR has the lowest computational complexity and the VA-SDR has the

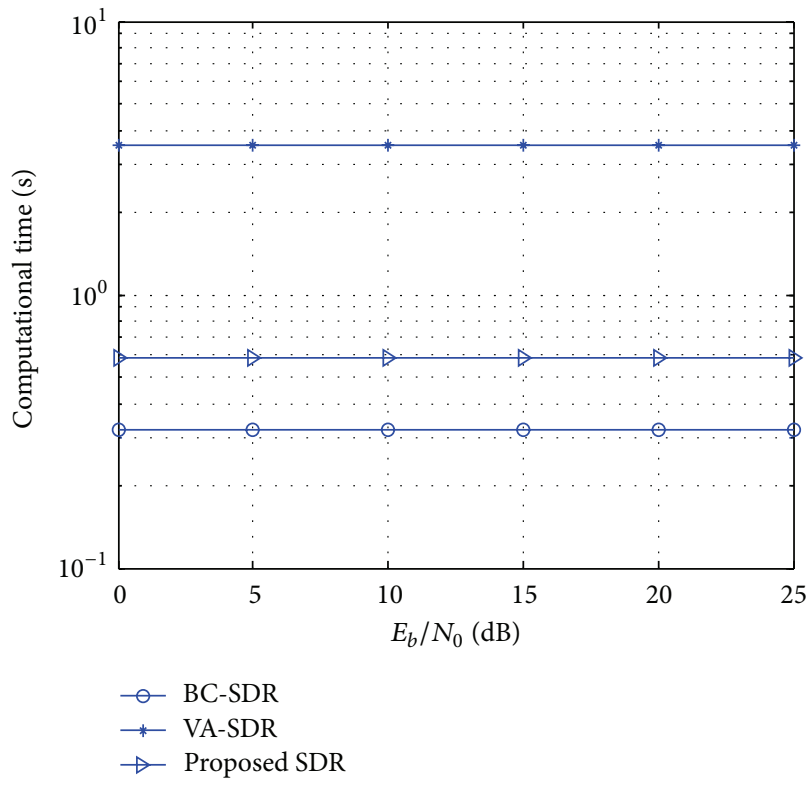

FIgURE 3: The computational time of the SDR detectors for $4 \times 4$ 256-QAM MIMO systems.

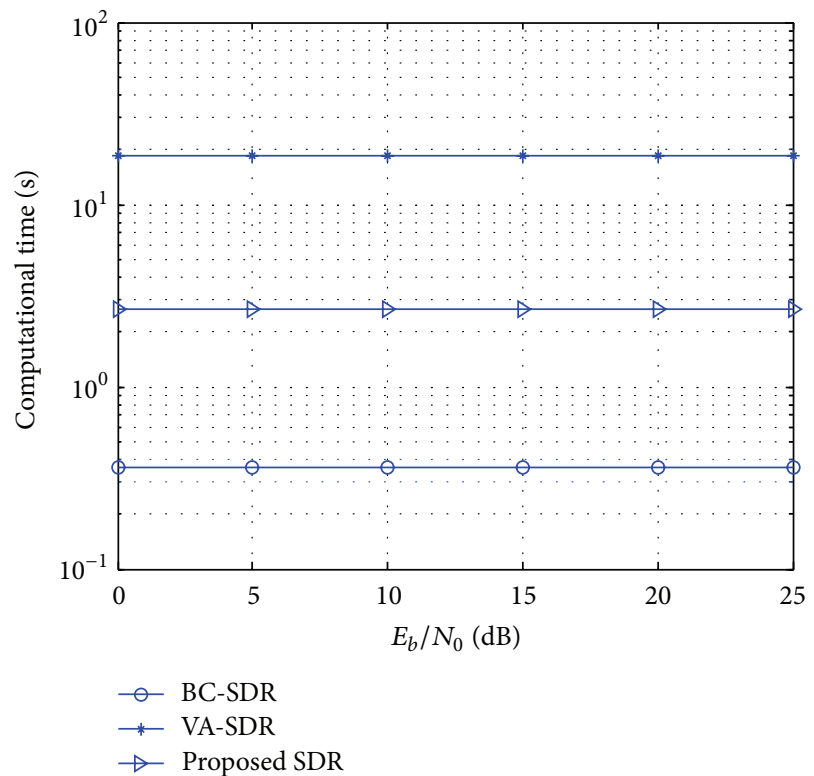

FIgURE 4: The computational time of the SDR detectors for $8 \times 8$ 256-QAM MIMO systems.

highest computational complexity, while the proposed SDR is in between.

\section{Conflict of Interests}

The authors declare that there is no conflict of interests regarding the publication of this paper. 


\section{References}

[1] J. Jaldén and B. Ottersten, "An exponential lower bound on the expected complexity of sphere decoding," in Proceedings of the IEEE International Conference on Acoustics, Speech, and Signal Processing (ICASSP '04), vol. 4, pp. iv-393-iv-396, Montreal, Canada, May 2004.

[2] A. Ben-Tal and A. Nemirovski, Lectures on Modern Convex Optimization, MPS-SIAM Series on Optimization, 2001.

[3] J. F. Sturm, "Using SEDUMI 1.02, A Matlab toolbox for optimization over symmetric cones," Optimization Methods and Software, vol. 11-12, pp. 1-30, 1999.

[4] B. Steingrimsson, Z. Q. Luo, and K. M. Wong, "Soft quasimaximum-likelihood detection for multiple-antenna wireless channels," IEEE Transactions on Signal Processing, vol. 51, no. 11, pp. 2710-2719, 2003.

[5] Z. Y. Shao, S. W. Cheung, and T. I. Yuk, "Lattice-reduction-aided semidefinite relaxation detection algorithms for multiple-input multiple-output systems," IET Communications, vol. 8, no. 4, pp. 448-454, 2014.

[6] W. K. Ma, T. N. Davidson, K. M. Wong, Z. Q. Luo, and P. C. Ching, "Quasi-maximum-likelihood multiuser detection using semi-definite relaxation with application to synchronous CDMA," IEEE Transactions on Signal Processing, vol. 50, no. 4, pp. 912-922, 2002.

[7] A. Wiesel, Y. C. Eldar, and S. Shamai, "Semidefinite relaxation for detection of 16-QAM signaling in MIMO channels," IEEE Signal Processing Letters, vol. 12, no. 9, pp. 653-656, 2005.

[8] P. H. Tan and L. K. Rasmussen, "The application of semidefinite programming for detection in CDMA," IEEE Journal on Selected Areas in Communications, vol. 19, no. 8, pp. 1442-1449, 2001.

[9] N. D. Sidiropoulos and Z.-Q. Luo, "A semidefinite relaxation approach to MIMO detection for high-order QAM constellations," IEEE Signal Processing Letters, vol. 13, no. 9, pp. 525-528, 2006.

[10] Z. Mao, X. Wang, and X. Wang, "Semidefinite programming relaxation approach for multiuser detection of QAM signals," IEEE Transactions on Wireless Communications, vol. 6, no. 12, pp. 4275-4279, 2007.

[11] W.-K. Ma, C.-C. Su, J. Jalden, T.-H. Chang, and C.-Y. Chi, "The equivalence of semidefinite relaxation MIMO detectors for higher-order QAM," IEEE Journal on Selected Topics in Signal Processing, vol. 3, no. 6, pp. 1038-1052, 2009.

[12] Z. Y. Shao, S. W. Cheung, and T. I. Yuk, "Semi-definite relaxation decoder for 256-QAM MIMO system," Electronics Letters, vol. 46, no. 11, pp. 796-797, 2010.

[13] Y. Yang, C. Zhao, P. Zhou, and W. Xu, "MIMO detection of 16QAM signaling based on semidefinite relaxation," IEEE Signal Processing Letters, vol. 14, no. 11, pp. 797-800, 2007.

[14] C. Helmberg, F. Rendl, R. J. Vanderbei, and H. Wolkowicz, "An interior-point method for semidefinite programming," SIAM Journal on Optimization, vol. 6, no. 2, pp. 342-361, 1996. 

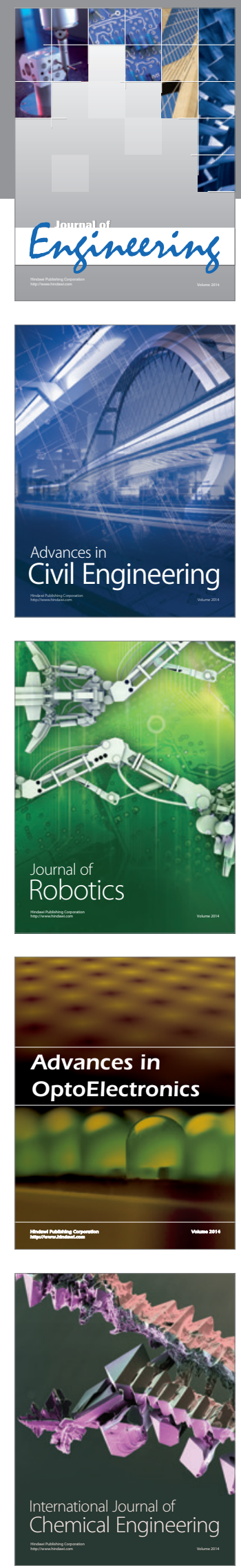

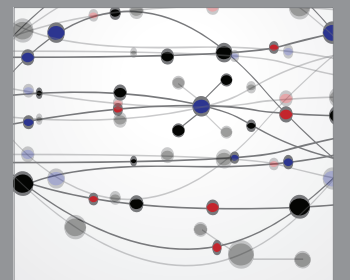

The Scientific World Journal
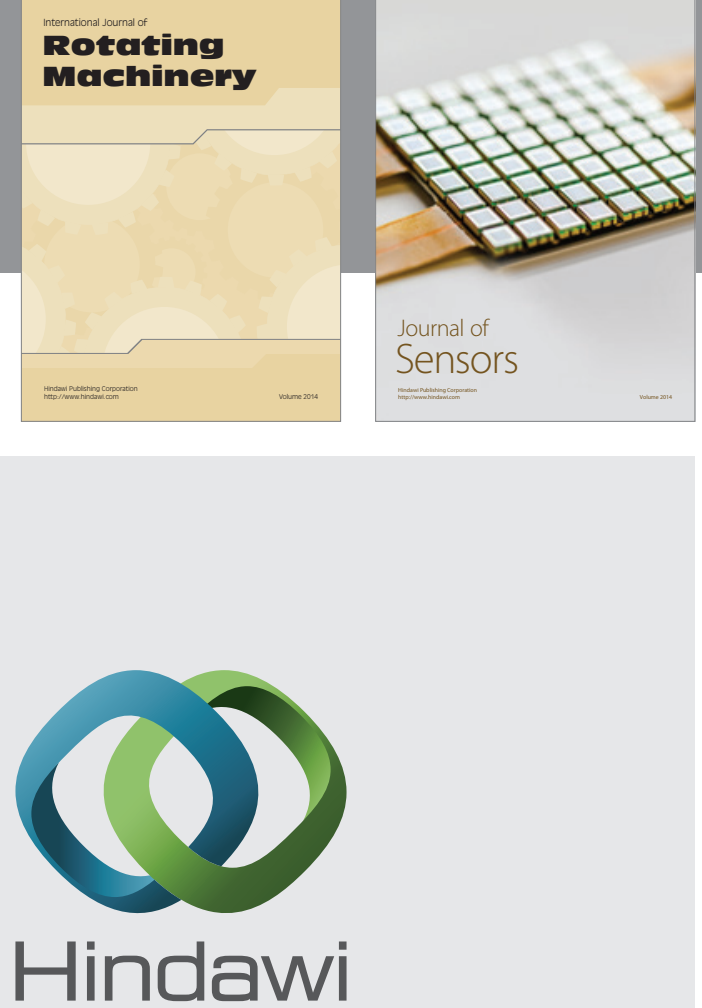

Submit your manuscripts at http://www.hindawi.com
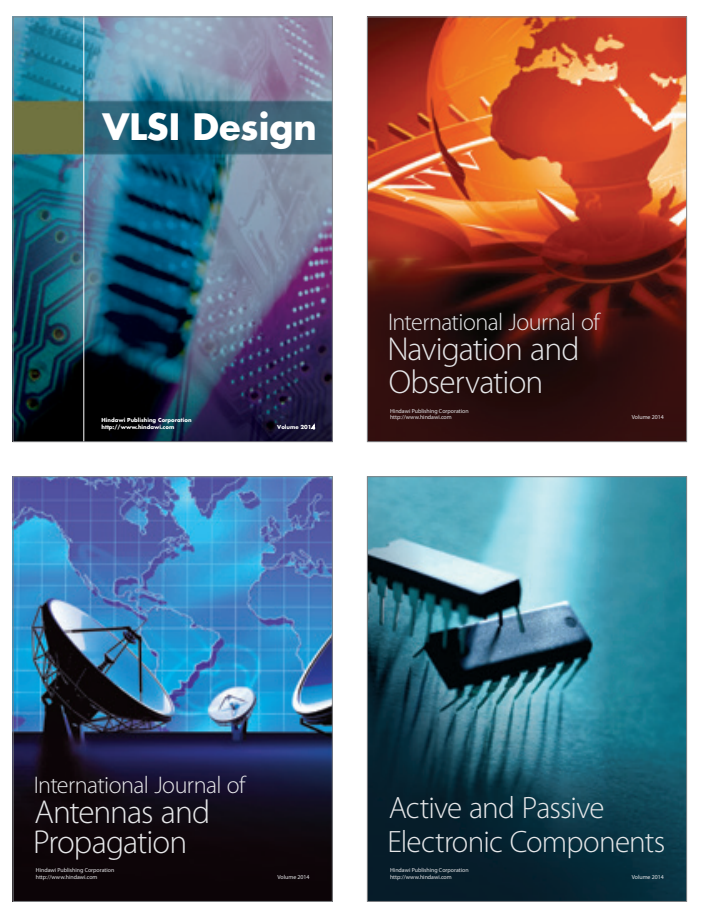
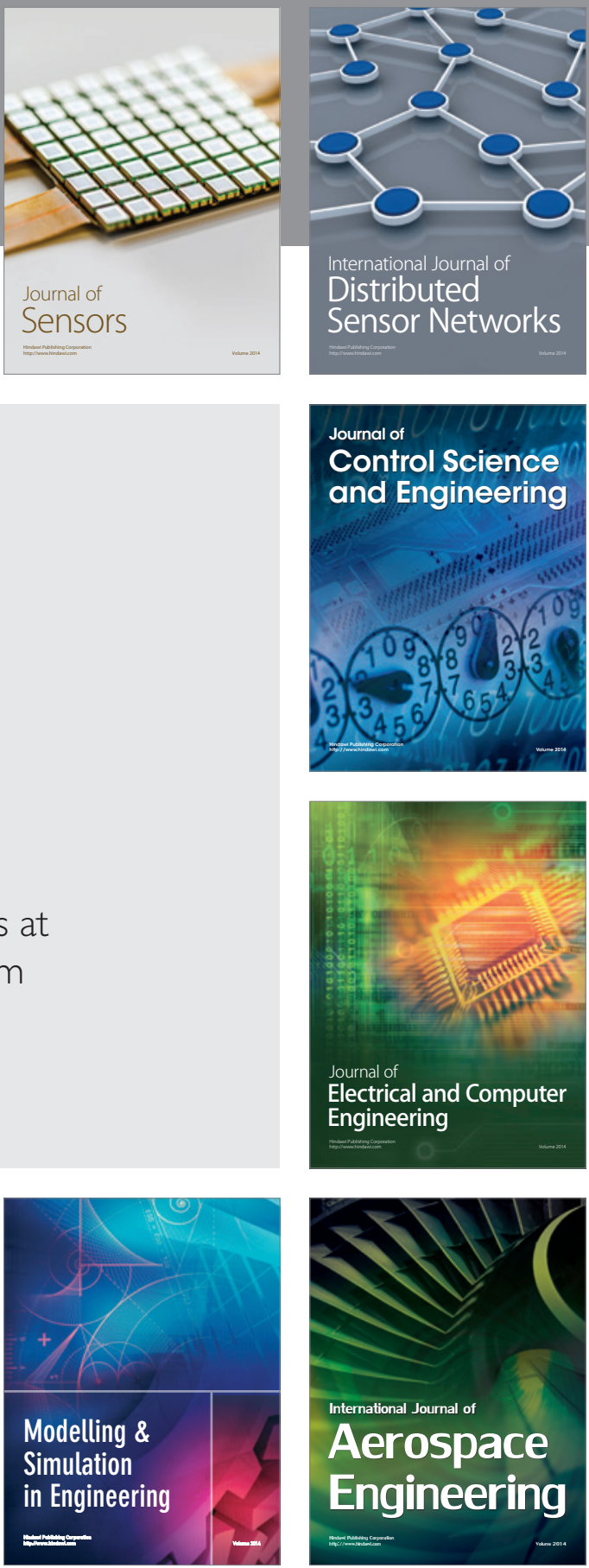

Journal of

Control Science

and Engineering
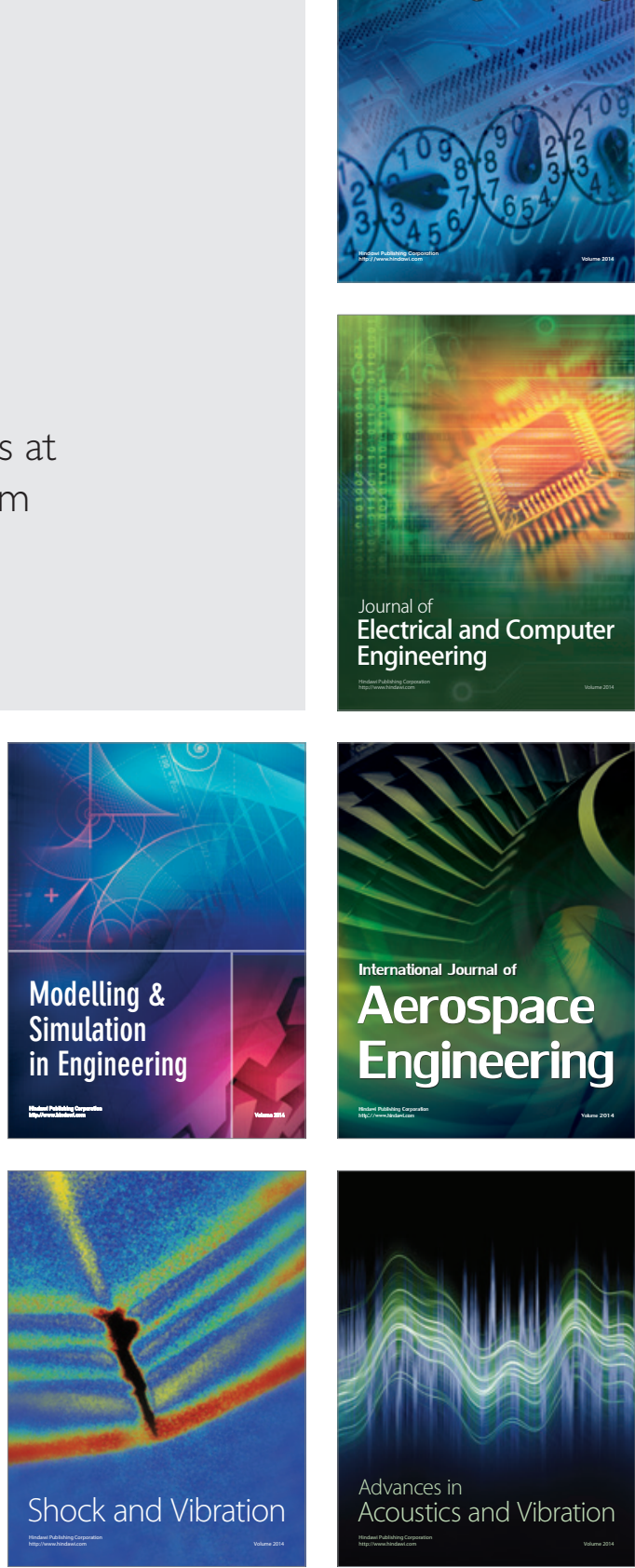